\title{
Tác động của danh tiếng tổ chức, tương thích con người - công việc, tương thích con người - tổ chức đến sự thu hút của tổ chức. Nghiên cứu trường hợp tại Bệnh viện Nhân dân Gia Định
}

\section{The impact of organizational reputation, person-job fit, and person-organization fit on organizational attraction. An empirical study at Gia Dinh People's Hospital}

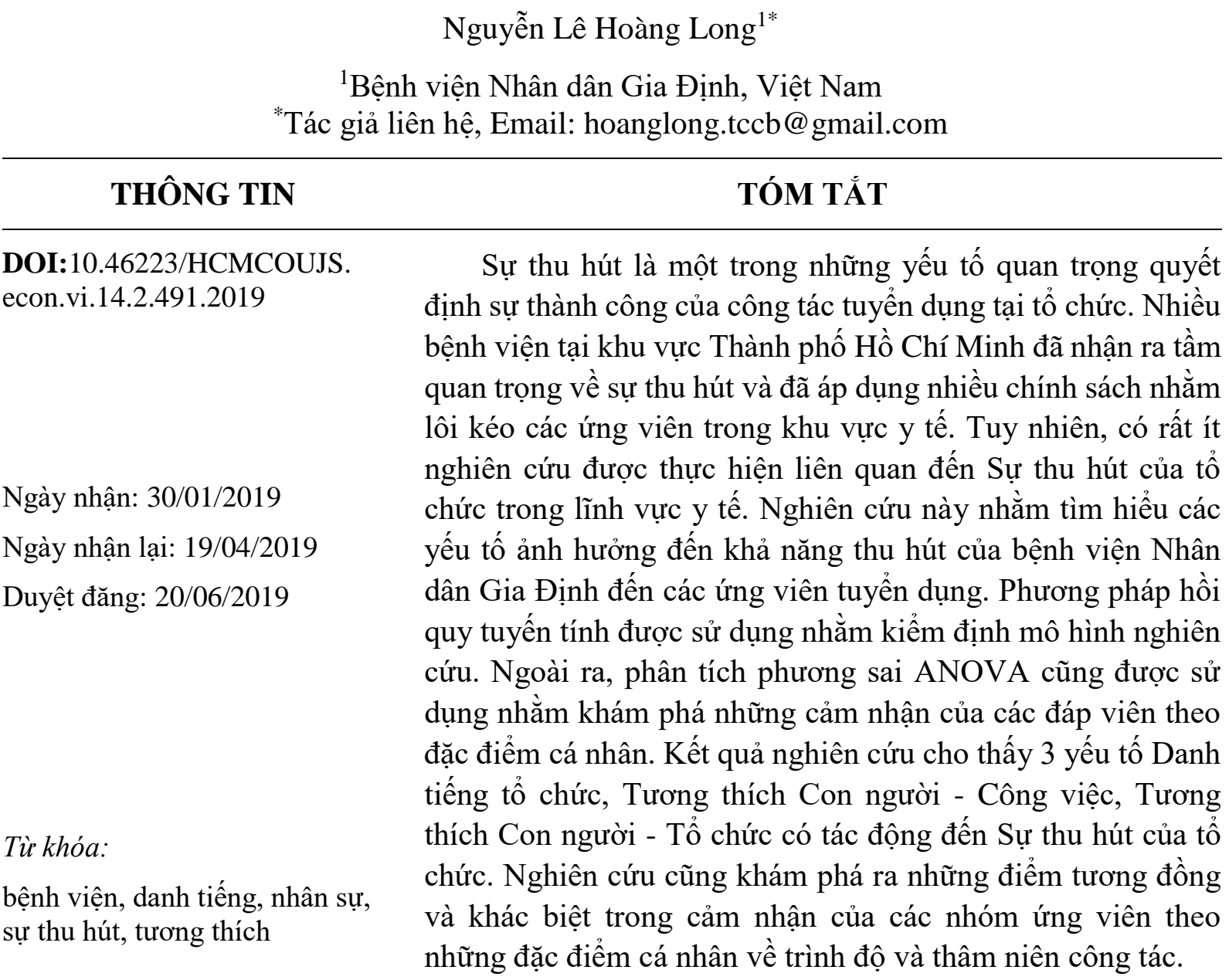

\begin{abstract}
Attractiveness is one of the important factors determining the success of recruitment at the organization. Many hospitals in Ho Chi Minh City have realized the importance of attractiveness and have adopted many policies to entice candidates in the health sector. However, there are very few studies done regarding the attractiveness of the organization at a public healthcare sector. This study aims to understand the
\end{abstract}


Keywords:

attraction, fit, hospital, human resource, reputation factors affecting the attractiveness of Gia Dinh People's Hospital to recruitment candidates. The linear regression method is used to verify the research model. In addition, ANOVA variance analysis is also used to explore the perceptions of respondents according to personal characteristics, the research results show that 3 factors of organizational reputation, person-job fit, and personorganization fit have an impact on organizational attraction. The study also explores the similarities and differences in the perception of candidates according to individual characteristics of qualifications and seniority.

\section{Mở đầu}

Sự cạnh tranh chất lượng dịch vụ công ngày càng trở nên gay gắt hơn trước những thay đổi trong nhu cầu của xã hội. Thêm vào đó, tình trạng chảy máu chất xám khiến cho rất nhiều đơn vị sự nghiệp công lập gặp khó khăn trong việc duy trì chất lượng dịch vụ mà điển hình là ngành y tế. Sự biến động nhân sự ở mức cao tạo ra nhiều thách thức cho các tổ chức ở nhiều khâu như đào tạo, hòa nhập nhân viên mới, ổn định chất lượng. Muốn khắc phục được tình trạng hiện tại, đòi hỏi công tác tuyển dụng cần phải được quan tâm hơn. Nghiên cứu gần đây cho thấy việc lựa chọn những ứng viên phù hợp với công việc có thể làm giảm đi khả năng nghỉ việc của họ sau này (Babakus, Yavas, \& Ashill, 2010; Liu, Liu, \& Hu, 2010). Công tác tuyển dụng muốn tìm được ứng viên có thể cống hiến lâu dài thì đòi hỏi phải đa dạng các khả năng lựa chọn của tổ chức, tạo được sự thu hút đối với những người đang tìm việc.

Sự thu hút của tổ chức là một khái niệm nhằm đánh giá sự hấp dẫn của tổ chức, khiến cho nhân viên muốn làm việc tại nơi đây (Van Hoye, 2012). Trong công tác tuyển dụng, sự thu hút có thể tạo cho các ứng viên hình thành nên ý định nộp đơn xin việc (Acarlar \& Bilgiç, 2013), dễ dàng chấp nhận đề nghị làm việc từ tổ chức (Carless, 2005), gia tăng sự cam kết với tổ chức (Mowday, Porter, \& Steers, 1982). Nhiều nghiên cứu trước đây đánh giá sự thu hút của tổ chức thông qua những yếu tố dễ dàng nhận biết được như mức lương (Honeycutt \& Rosen, 1997) hay khả năng ứng xử của chuyên viên thực hiện phỏng vấn tuyển dụng (Carless \& Wintle, 2007).

Tuy nhiên, sự thu hút của tổ chức đối với một ứng viên còn có thể được tạo ra từ các yếu tố vô hình như niềm say mê vị trí công việc dự tuyển của ứng viên đó (Carless, 2005) hoặc đến từ cảm nhận của ứng viên cảm thấy mình phù hợp với bản thân tổ chức này (Judge \& Cable, 1997); một yếu tố cũng tạo nên Sự thu hút của tổ chức đối với ứng viên chính là bản thân danh tiếng của tổ chức (Turban \& Cable, 2003).

Nghiên cứu này tìm hiểu cảm nhận bị thu hút của ứng viên xin việc trong trường hợp cụ thể tại bệnh viện Nhân dân Gia Định. Về mặt học thuật, nghiên cứu đóng góp thêm một bối cảnh mới tại khu vực y tế công ở Việt Nam mà chưa có nghiên cứu nào được thực hiện trước đó. Ngoài ra, nghiên cứu thực hiện so sánh liệu cảm nhận về sự tương thích của ứng viên đối với tổ chức tuyển dụng sẽ khiến họ bị hấp dẫn hay do chính bản thân danh tiếng của tổ chức đủ để tạo ra sự thu hút. Cuối cùng, nghiên cứu nêu lên một số hàm ý chính sách nhằm nâng cao chất lượng công tác tuyển dụng tại Bệnh viện Nhân dân Gia Định. 


\section{Cơ sở lý thuyết và giả thuyết nghiên cứu}

\subsection{Danh tiếng tổ chức}

Danh tiếng tổ chức được hiểu là những đánh giá chung về một tổ chức thông qua khả năng mang đến những giá trị cho một nhóm đại diện hay các bên liên quan (Fombrun, Gardberg, $\&$ Sever, 2000). Danh tiếng tổ chức tạo ra ảnh hưởng trong quyết định của người lao động trong việc nộp đơn và một vị trí ứng tuyển (Cable \& Turban, 2003). Danh tiếng tổ chức còn được định nghĩa rộng hơn là một tập hợp về niềm tin về tổ chức trên các phương diện liên quan đến năng lực, lịch sử, sứ mệnh của tổ chức đó (Carpenter, 2010).

\subsection{Tưong thích Con người - Công việc}

Sự tương thích Con người - Công việc là nền tảng trong việc lựa chọn nhân viên trong công tác tuyển dụng của tổ chức (Werbel \& Gilliland, 1999). Tương thích giữa con người và công việc chính là những yêu cầu của vị trí việc làm về kỹ năng, kiến thức, và những năng lực khác trong thực hiện công việc phù hợp với khả năng của nhân viên đến mức độ như thế nào (Taylor, 1911).

\subsection{Tương thích Con ngưòi - Tổ chức}

Những tương đồng giữa các giá trị và quy tắc của tổ chức và nhân viên được định nghĩa là sự tương thích Con người - Tổ chức (Chatman, 1989). Kristof (1996) định nghĩa Tương thích Con người - Tổ chức xuất hiện khi một bên thỏa mãn nhu cầu cho bên còn lại hay giữa Con người và Tổ chức cùng chia sẻ những đặc điểm tương đồng hoặc là cả hai khả năng này xảy ra cùng một lúc.

\subsection{Sụ thu hút của tổ chức}

Sự thu hút tổ chức được xem là yếu tố quan trọng ảnh hưởng đến hành vi lựa chọn công việc của cá nhân (Rynes, Bretz, \& Gerhart, 1991). Sự thu hút của tổ chức đối với cá nhân được biểu hiện trên nhiều phương diện như: Hành vi tổ chức, công tác quản trị nguồn nhân lực, chiến lược phát triển của tổ chức (Perez, Walton, Cooper, \& Pacheco, 2014). Sự thu hút của tổ chức được chứng minh có tương quan tích cực đến kết quả đầu ra của tổ chức như cải thiện hiệu suất, giảm biến động trong công tác nhân sự (Kristof-Brown, Zimmerman, \& Johnson, 2005).

\subsection{Lập luận giả thuyết}

\subsubsection{Danh tiếng tổ chức và Sự thu hút của tổ chức}

Lawler, Kuleck, Rhode, và Sorensen (1975) nghiên cứu trên 431 sinh viên ngành kế toán cho thấy Danh tiếng tổ chức có tác động dương trực tiếp đến Sự thu hút của tổ chức. Một nghiên cứu khác dựa trên các đối tượng đang tìm việc của Cable và Turban (2003) đã khám phá ra rằng ngoài việc công ty có danh tiếng sẽ có lợi thế trong thu hút ứng viên dự tuyển thì những công ty này có cơ hội cao hơn trong việc lựa chọn được những ứng viên có chất lượng. Nghiên cứu rộng hơn của Collins và Han (2004) sử dụng dữ liệu thu thập được từ 99 tổ chức khác nhau cũng khẳng định được mối liên hệ mạnh mẽ giữa Danh tiếng tổ chức đến số lượng và chất lượng ứng viên dự tuyển.

Từ đó ta có giả thuyết:

H1: Danh tiếng tổ chức tác động dưong đến Sụ thu hưt của tổ chức 


\subsubsection{Tương thích Con ngườ - Công việc và Sụ thu hút của tổ chức}

Carless (2005) khi nghiên cứu về Sự thu hút của tổ chức và Ý định chấp thuận nhận việc của 193 ứng viên thông qua 3 thời điểm trước, trong và sau khi phỏng vấn đã cho thấy tác động ảnh hưởng cùng chiều của sự Tương thích Con người - Tổ chức đến Sự thu hút của tổ chức. Nghiên cứu còn chỉ ra rằng sự tương thích với công việc ứng tuyển không chỉ thu hút ứng viên đến với tổ chức mà còn thúc đẩy ý định chấp nhận lời đề nghị từ tổ chức. Christensen và Wright (2011) còn phát hiện thêm những nhân viên có sự tương thích với tổ chức sẽ bị hấp dẫn hơn bởi tổ chức và xuất hiện động lực phụng sự công (public service motivation) cao hơn những nhân viên không tương thích với công việc, nghiên cứu của Christensen và Wright (2011) là quan trọng trong khu vực công vì động lực phụng sự công sẽ tạo ra nhiều hành vi có lợi trong thực hiện sứ mạng công của tổ chức.

Từ đó ta có giả thuyết:

\section{H2: Tương thích Con người - Công việc tác động dương đến Sự thu hút của tổ chưc}

\subsubsection{Tương thích Con ngườ - Tổ chức và Sụ thu hút của tổ chức}

Tương thích Con người - Tổ chức có ảnh hưởng đến quyết định của nhà tuyển dụng khi lựa chọn ứng viên (Cable \& Judge, 1997) và ứng viên khi lựa chọn làm việc cho tổ chức (Cable \& Judge, 1996). Nghiên cứu của Dineen, Ash, và Noe (2002) kết luận sự tương thích đối với tổ chức về những mục tiêu hay sự tôn trọng lẫn nhau sẽ tác động dương đến Sự thu hút của tổ chức. Cable và Judge (1996) cho thấy Tương thích Con người - Tổ chức ảnh hưởng đến thái độ và ý định của những ứng viên tuyển dụng.

Từ đó ta có giả thuyết:

H3: Tương thích Con người - Tổ chức tác động dương đến Sự thu hút của tổ chức

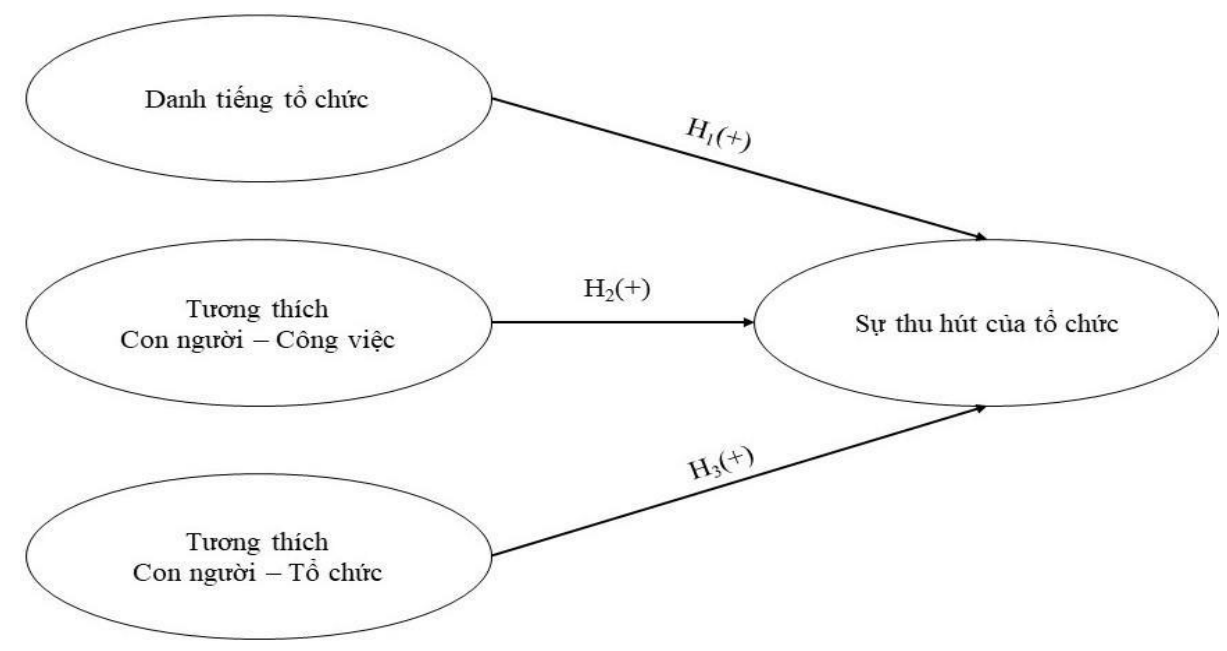

Hình 1. Mô hình nghiên cứu đề xuất

\section{Thiết kế nghiên cứu}

\subsection{Thang đo và mã hoá}

Danh tiếng tổ chúc:

Nghiên cứu này sử dụng thang đo của Tạp chí Fortune về danh tiếng tiếng tổ chức 
(1993). Thang đo gồm 5 biến quan sát: Tổ chức nổi tiếng về chất lượng quản lý (OR1); Tổ chức nổi tiếng về chất lương dịch vu (OR2); Tổ chức nổi tiếng về các dụ án đầu tu dài hạn (OR3); Tổ chức nổi tiếng về khả năng thu hút, phát triển và giữ chân người tài (OR4); Tổ chíc nổi tiếng về trách nhiệm đối với cộng đồng (OR5).

\section{Tuơng thích Con người - Công việc}

Sự phù hợp giữa cá nhân và công việc sử dụng thang đo từ nghiên cứu của Saks và Ashforth (1997) bao gồm 4 biến quan sát: Kiến thức, kỹ năng phù hơp với công việc ứng tuyển (PJ1); Vị trí công việc đáp ứng nhu cầu cá nhân (PJ2); Bản thân công việc thực sự phù hợp với cá nhân (PJ3); Công việc cho phép cá nhân thực hiện những gì muốn làm (PJ4).

\section{Tương thích Con ngưò̀i - Tổ chức}

Thang đo của Judge và Cable (1997) được sử dụng để đo lường sự phù hợp của con người và tổ chức bao gồm 4 biến quan sát: Nhũng giá trị của tổ chức tuoơng đồng với cá nhân (PO1); Tính cách cá nhân phù hợp với cá tính hoặc hình ảnh của tổ chức (PO2); Tổ chức đáp ưng được nhu cầu của cá nhân (PO3); Bản thân tổ chức là phù hợp với cá nhân (PO4).

\section{Sụ thu hút của tổ chức}

Sự thu hút của tổ chức đo lường bằng thang đo của Smither, Reilly, Millsap, Pearlman, và Stoffey (1993). Thang đo gồm 3 biển quan sát: Tổ chức này là một trong những nơ tốt nhất để làm việc (OT1); Nhìn chung mức thu nhập tại tổ chức là tốt (OT2); Có nhiều co hội thăng tiến khi làm việc tại tổ chức (OT3).

\subsection{Phưong pháp nghiên cứu}

\subsubsection{Nghiên cứu định tính}

Nghiên cứu định tính được tiến hành nhằm hiệu chỉnh các biến quan sát không phù hợp với địa điểm nghiên cứu cũng như hiệu chỉnh từ ngữ trong bảng hỏi thông qua việc tham khảo ý kiến người làm công tác tuyển dụng tại Bệnh viện Nhân dân Gia Định và các ứng viên nộp hồ sơ xin việc tại đây.

\subsubsection{Nghiên cúu định lượg}

Nghiên cứu sử dụng phần mềm SPSS 23 nhằm kiểm định các nội dung nghiên cứu như phân tích thống kê mô tả, đánh giá độ tin cậy thang đo bằng hệ số Cronbach's Alpha, phân tích nhân tố khám phá EFA, phân tích hồi quy tuyến tính và phân tích phương sai ANOVA.

\section{Kết quả nghiên cứu và bình luận}

\subsection{Mô tả mẫu nghiên cứu}

Nghiên cứu sử dụng nguồn dữ liệu sơ cấp chính các ứng viên dự tuyển tại bệnh viện Nhân dân Gia Định từ tháng 09/2018 đến tháng 01/2019. Các ứng viên được phát bảng hỏi hoàn toàn là ngẫu nhiên và hoàn toàn phù hợp với đối tượng nghiên cứu. Dữ liệu được khảo sát đúng đối tượng và mang tính khách quan sẽ phản ánh được kết quả nghiên cứu phù hợp, có giá trị khoa học và thuận lợi cho việc mở rộng hướng nghiên cứu (Neuman, 2007). Khảo sát bao gồm việc khảo sát trực tiếp các ứng viên sau phỏng vấn và gửi email khảo sát thông qua công cụ Google Form đối với các ứng viên bỏ về ngay sau phỏng vấn, không thể tiếp xúc trực tiếp. Số lượng email đã gửi khảo sát là 67 , Số lượng ứng viên đã khảo sát trực tiếp là 78 . Tổng số bảng 
khảo sát đã nhận về là 145, sau khi xử lý, loại bỏ các phiếu không hợp lệ và làm sạch dữ liệu là 131.

Trong số các phiếu trả lời hợp lệ, tỷ lệ về trình độ học vấn là tương đồng nhau. Cụ thể, ứng viên có trình độ học vấn Trung cấp là $29 \%$, Cao đẳng là $18.3 \%$, Đại học là $23.7 \%$, và sau Đại học là $29 \%$. Thâm niên công tác của các ứng viên phân bổ phần lớn vào các ứng viên có kinh nghiệm công tác dưới 5 năm. Cụ thể, ứng viên chưa từng đi làm là $22.9 \%$, thâm niên dưới 1 năm $26 \%$, thâm niên từ 1 đến dưới 3 năm là $13 \%$, thâm niên từ 3 đến dưới 5 năm là $20.6 \%$, và thâm niên trên 5 năm là $17.6 \%$.

\subsection{Kiểm định thang đo}

Hệ số Cronbach's Alpha được thực hiện đối với mỗi nhóm biến cố kết thành các yếu tố. Do đây là nghiên cứu mới lấy bối cảnh tại bệnh viện Nhân dân Gia Định vì vậy các biến quan sát có hệ số tương quan biến - tổng nhỏ hơn 0,3 sẽ bị loại bỏ và tiêu chuẩn để chọn thang đo khi có độ tin cậy alpha từ 0,6 trở lên (Hair, Black, Babin, Anderson, \& Tatham, 2006), các nhóm biến cố có hệ số Cronbach's Alpha quá lớn $(>0.95)$ cũng sẽ được xem xét hiện tượng trùng lắp trong đo lường (Nguyen, 2012). Sau khi kiểm định, tất cả các biến quan sát đều đạt yêu cầu.

Phân tích nhân tố khám phá EFA được sử dụng nhằm phân tích tương quan giữa các biến với nhau và rút thành một tập hợp các yếu tố có ý nghĩa. Trong nghiên cứu này sử dụng phép trích nhân tố Principal Component với phép quay vuông góc Varimax cho 2 nhóm biến độc lập và phụ thuộc. Kết quả cho thấy các biến quan sát đều hội tụ về đúng nhân tố đo lường và có hệ số tải nhân tố $>0.5$. Kết quả rút trích được 3 nhóm biến độc lập bao gồm: Danh tiếng tổ chức (OR) gồm 5 biến quan sát; Tưong thích Con nguời - Công việc (PJ) gồm 4 biến quan sát; Tương thích Con ngườ - Tổ chưc (PO) gồm 4 biến quan sát. Một nhóm biến phụ thuộc được rút trích là Sự thu hút của tổ chức (OT) gồm 3 biến quan sát.

\section{Bảng 1}

Kết quả kiểm định thang đo

\begin{tabular}{|c|c|c|c|c|c|}
\hline Nhóm biến cố & $\begin{array}{l}\text { Số biến } \\
\text { quan sát }\end{array}$ & $\begin{array}{c}\text { Giá trị } \\
\text { Cronbach's } \\
\text { Alpha }\end{array}$ & $\begin{array}{l}\text { Hệ số tải } \\
\text { nhân tố }\end{array}$ & $\begin{array}{c}\text { Kiểm định KMO/ } \\
\text { Bartlett }\end{array}$ & $\begin{array}{l}\text { Phương } \\
\text { sai trích }\end{array}$ \\
\hline Danh tiếng tổ chức & 5 & 0.792 & $\begin{array}{l}0.753 ; \\
0.747 \\
0.641 \\
0.628 \\
0.601\end{array}$ & \multirow{3}{*}{$\begin{array}{c}\mathrm{KMO}=0.888>0.5 \\
\text { P.value }=0.000<0.05\end{array}$} & \multirow{3}{*}{$56.246 \%$} \\
\hline $\begin{array}{l}\text { Tương thích Con } \\
\text { người - Công việc }\end{array}$ & 4 & 0.697 & $\begin{array}{l}0.750 \\
0.680 \\
0.608 \\
0.583\end{array}$ & & \\
\hline $\begin{array}{l}\text { Tương thích Con } \\
\text { người - Tổ chức }\end{array}$ & 4 & 0.755 & $\begin{array}{l}0.785 \\
0.732 \\
0.684\end{array}$ & & \\
\hline
\end{tabular}




\begin{tabular}{|c|c|c|c|c|c|}
\hline Nhóm biến cố & $\begin{array}{c}\text { Số biến } \\
\text { quan sát }\end{array}$ & $\begin{array}{c}\text { Giá trị } \\
\text { Cronbach's } \\
\text { Alpha }\end{array}$ & $\begin{array}{c}\text { Hệ số tải } \\
\text { nhân tố }\end{array}$ & $\begin{array}{c}\text { Kiểm định KMO/ } \\
\text { Bartlett }\end{array}$ & $\begin{array}{c}\text { Phương } \\
\text { sai trích }\end{array}$ \\
\hline & & & 0.630 & & \\
\hline $\begin{array}{c}\text { Sự thu hút của tổ } \\
\text { chức }\end{array}$ & 3 & 0.871 & $\begin{array}{c}0.922 ; \\
0.889 ; \\
0.867\end{array}$ & $\begin{array}{c}\text { KMO }=0.719>0.5 \\
\text { P.value }=0.000<0.05\end{array}$ & $79.732 \%$ \\
\hline
\end{tabular}

Nguồn: Kết quả xử lý từ dữ liệu điều tra

\subsection{Phân tích hồi quy tuyến tính bội}

Phương pháp Stepwise được sử dụng trong việc ước lượng mô hình hồi quy. Kết quả phân tích tại Bảng 2 với mô hình đầu tiên nhận giá trị $\mathrm{R}^{2}$ hiệu chỉnh là $0.425(<0.5)$ nghĩa là $42.5 \%$ Sự thu hút của tổ chức được giải thích bởi mối quan hệ tuyến tính với danh tiếng tổ chức. Kết quả này chưa phản ánh đầy đủ được mối liên hệ giữa Sự thu hút của tổ chức với các yếu tố khác và do đó còn bỏ sót những yếu tố có khả năng tác động.

Tại mô hình hồi quy thứ 2 khám phá mối quan hệ tuyến tính giữa Danh tiếng tổ chức, Tương thích con người - Tổ chức đối với Sự thu hút của tổ chức. Kết quả phân tích hồi quy với mô hình thứ 2 thể hiện kết quả $\mathrm{R}^{2}$ hiệu chỉnh xấp xỉ đạt yêu cầu $(0.4970 .5)$. Tuy nhiên, sự tương thích cần phải được đánh giá ở cả 2 mặt công việc lẫn bản thân tổ chức. Do đó, mô hình hồi quy thứ 2 thiếu vắng sự phù hợp của con người đối với công việc và vẫn có thể chưa đạt kết quả tối ưu nhất.

Mô hình hồi quy thứ 3 khảo sát trọn vẹn mối quan hệ tuyến tính giữa Danh tiếng tổ chức và cảm nhận tương thích của ứng viên về công việc lẫn tổ chức tuyển dụng. Kết quả phân tích hồi quy tuyến tính bội tại Bảng 2 cho thấy hệ số $\mathrm{R}^{2}$ hiệu chỉnh đạt giá trị $0,515(>0.5)$ nghĩa là $51.5 \%$ sự biến thiên đối với Sự thu hút của tổ chức được giải thích bởi mối quan hệ tuyến tính của các biến độc lập. Thông qua so sánh hệ số $\mathrm{R}^{2}$ hiệu chỉnh ta có thể thấy được mô hình hồi quy với đầy đủ các biến giống với mô hình nghiên cứu đề xuất là đạt kết quả tối ưu nhất tại nghiên cứu này. Bảng 2 cũng cho thấy rằng kiểm định thống kê $\mathrm{F}$ có mức ý nghĩa Sig. $=0.000$ nên ta có thể bác bỏ giả thuyết $\mathrm{H} 0: \mathrm{R}^{2}=0$, kết luận rằng mô hình hồi quy tuyến tính bội được xây dựng phù hợp.

Dò tìm các phạm vi giả định cần thiết về tính độc lập của sai số bằng thực hiện lấy giá trị Durbin - Watson. Giá trị nhận được là 1.612 nằm trong khoảng chấp nhận được $(<2)$ nên không có hiện tượng tương quan giữa các phần dư trong mô hình. Kiểm tra hiện tượng đa cộng tuyến bằng hệ số phóng đại phương sai VIF cho thấy các biến độc lập đều thỏa yêu cầu $(<10)$ do đó không xuất hiện hiện tượng đa cộng tuyến. 


\section{Bảng 2}

Kết quả phân tích hồi quy tuyến tính bội

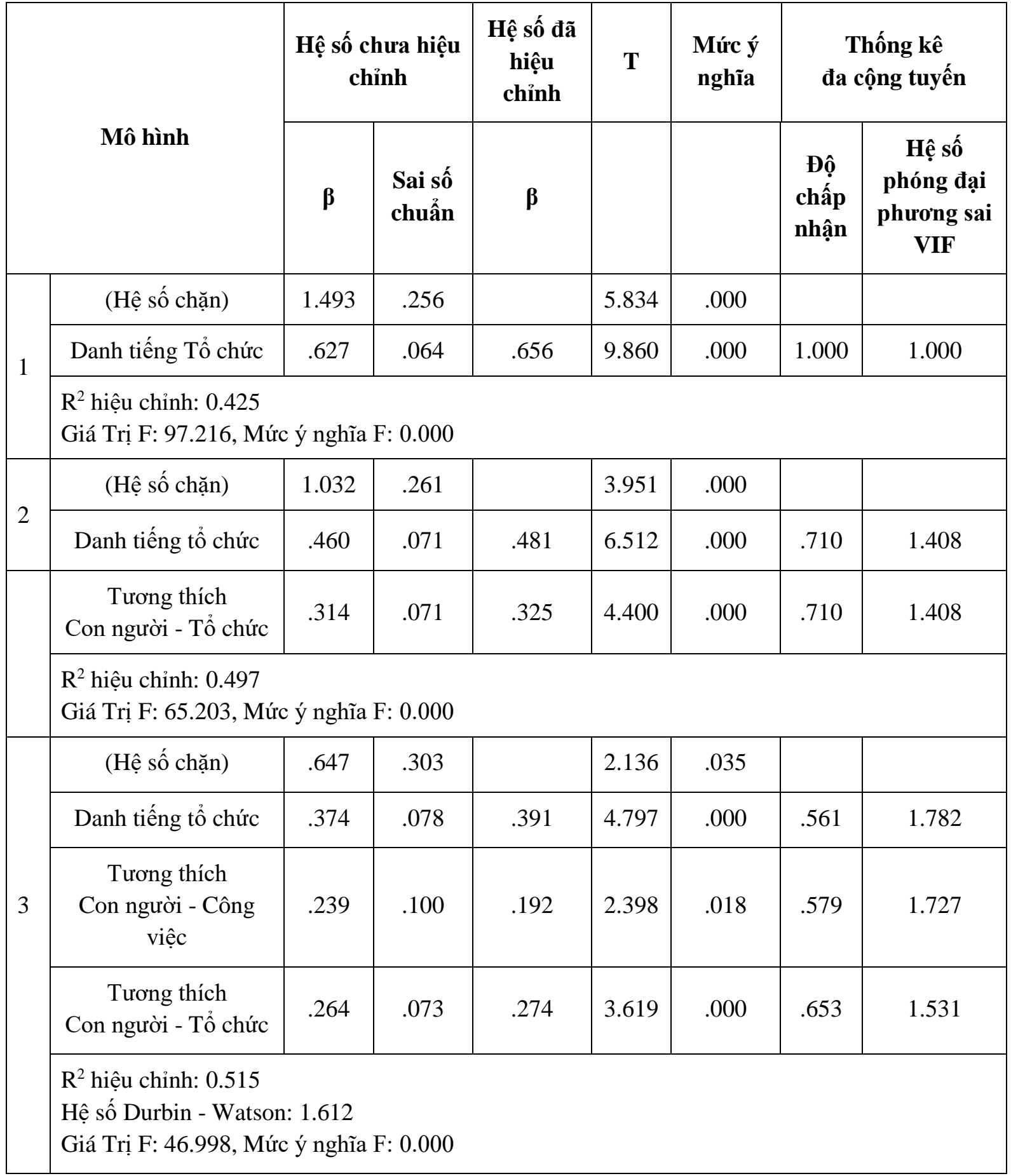

Nguồn: Kết quả xử lý từ dữ liệu điều tra

Kết quả phân tích hồi quy tuyến tính bội cho thấy Danh tiếng tổ chức, Tương thích Con người - Công việc, Tương thích Con người - Tổ chức đều tác động dương đến Sự thu hút của tổ chức với mức ý nghĩa Sig. <0.05. Tất cả hệ số $\beta$ của 3 biến độc lập đều là số dương, phản 
ánh tác động cùng chiều của biến độc lập và biến phụ thuộc. Điều này hoàn toàn phù hợp với các giả thuyết nghiên cứu.

Với mức ý nghĩa Sig. $=0.000(<0.05)$ và hệ số $\beta=0.391$, chấp nhận giả thuyết $\mathrm{H}_{1}$ : Danh tiếng tổ chức tác động dương đến Sự thu hút của tổ chức. Trong thực tế, dễ dàng nhận thấy sự phổ biến của một tổ chức sẽ tạo ra một ấn tượng nhất định đến các nhân vật hữu quan bên ngoài tổ chức, trong đó có các ứng viên tuyển dụng. Các ứng viên khi tiến hành ứng tuyển tại một tổ chức thì đều có xu hướng tìm hiểu thông tin về tổ chức và chính độ phổ biến, sự nổi tiếng của tổ chức sẽ tạo ra những thông tin đến từ gia đình, xã hội, các kênh thông tin truyền thông và là một nguồn thông tin tham khảo có giá trị ảnh hưởng đển cảm nhận lôi cuốn của các ứng viên.

Với mức ý nghĩa Sig. $=0.018(<0.05)$ và hệ số $\beta=0.192$, chấp nhận giả thuyết $\mathrm{H}_{2}$ : Tương thích Con người - Công việc tác động dương đến Sự thu hút của tổ chức. Trong thực tế, sự phù hợp của một cá nhân với công việc ứng tuyển sẽ tạo được sự hấp dẫn đối với ứng viên tuyển dụng. Chính những sự tương thích về kỹ năng, quan điểm, các giá trị liên quan đển công việc đã hình thành nên một lực hẩp dẫn của tổ chức đối với các ứng viên xin việc tại đây. Trong lĩnh vực y tế, sự tương thích giữa con người và công việc là vô cùng quan trọng, ngoài kiến thức chuyên môn thì tấm lòng của nhân viên y tế trong phục vụ bệnh nhân cũng sẽ góp phần làm gia tăng chất lượng dịch vụ khám chữa bệnh. Người nhân viên y tế hội đủ kiến thức chuyên môn lẫn sự đam mê trong công việc sẽ cảm thấy phù hợp với vị trí việc làm được tuyển dụng, dẫn đến niềm yêu thích của họ đến với tổ chức y tế mà cá nhân thực hiện ứng tuyển.

Với mức ý nghĩa Sig. $=0.000(<0.05)$ và hệ số $\beta=0.274$, chấp nhận giả thuyết $\mathrm{H}_{3}$ : Tương thích Con người - Tổ chức tác động dương đến Sự thu hút của tổ chức. Sự tương thích với tổ chức sẽ thúc đẩy quá trình hòa nhập nhân viên mới đến với tổ chức, tạo ra suy nghĩ tốt của nhân viên đến với tổ chức tuyển dụng, dẫn đến hình thành Sự thu hút của tổ chức đến với ứng viên. Ứng viên ngoài việc phải phù hợp với công việc thì cũng cần đánh giá xem họ có thể làm việc tại tổ chức này hay không, Hiện tại, nhiều cơ sở y tế công cố gắng gia tăng sự tương thích Con người - Tổ chức để thu hút các ứng viên tuyển dụng thông qua các chế độ, chính sách ưu đãi bao gồm lương, thưởng, đào tạo trong và ngoài nước, các khoản phụ cấp ưu đãi nghề và nhiều chế độ khác.

Kết quả hồi quy tuyến tính bội đã chỉ ra mức độ tác động khác nhau của Danh tiếng tổ chức (0.391), Tương thích Con người - công việc (0.192), Tương thích Con người - Tổ chức (0.274). Danh tiếng tổ chức chính là yếu tố tác động mạnh nhất khiến cho các ứng viên tuyển dụng bị thu hút bởi tổ chức. Tương thích Con người - Tổ chức có tác động thấp hơn danh tiếng của tổ chức trong việc tạo ra sự hấp dẫn các ứng viên. Sự phù hợp với công việc ứng tuyển cũng có tác động đến khả năng lôi cuốn các ứng viên. Tuy nhiên, dường như trong công tác tuyển dụng chưa thật sự truyền đạt trọn vẹn cho các ứng viên hiểu được vai trò cũng như nhiệm vụ sẽ chờ đợi họ trong tương lai. Vì vậy, tác động của của Sự tương thích Con người - công việc đến Sự thu hút của tổ chức là thấp hơn hẳn so với 2 yếu tố trên.

\subsection{Phân tích ANOVA}

4.4.1. Phân tích ảnh hưởng của trình độ học vấn đến Sự thu hút của tổ chức bằng phuoong pháp phân tích phuoong sai ANOVA

Ngoài việc phân tích hồi quy tuyến tính bội, nghiên cứu cũng xem xét sự khác biệt về cảm nhận của các ứng viên theo các đặc điểm cá nhân. Kết quả phân tích nhận giá trị Sig. > 
$0.05(0.708)$ tại kiểm định Homogeneity do đó có thể có thể tiến hành theo dõi kết quả phân tích ANOVA.

Tại kiểm định ANOVA cho các nhóm trình độ học vấn bao gồm: Trung cấp, Cao đẳng, Đại học, Sau đại học; ta nhận được giá trị Sig. $>0.05$ (0.233) do đó có thể kết luận rằng không có sự khác biệt có ý nghĩa thống kê trong cảm nhận giữa các nhóm ứng viên có trình độ học vấn khác nhau về Sự thu hút của tổ chức đối với họ, hay nói cách khác thì cảm nhận về tính hấp dẫn của tổ chức đối các nhóm ứng viên tuyển dụng có trình độ học vấn khác nhau là như nhau.

4.4.2. Phân tích ảnh hương của thâm niên công tác đến Sự thu hút của tổ chức bằng phuoong pháp phân tích phioong sai ANOVA

Tiến hành kiểm định cảm nhận giữa các nhóm nhân viên có thâm niên công tác khác nhau đến Sự thu hút của tổ chức. Kết quả phân tích tại kiểm định Homogeneity nhận giá trị Sig. $>0.05(0.245)$ do đó có thể tiến hành theo dõi kết quả phân tích ANOVA.

Tại kiểm định ANOVA cho các nhóm ứng viên có thâm niên công tác bao gồm: Chưa từng đi làm, Thâm niên dưới 1 năm, Thâm niên từ 1 đến dưới 3 năm, Thâm niên từ 3 năm đến dưới 5 năm, Thâm niên trên 5 năm; ta nhận được giá trị Sig. $<0.05(0.000)$ do đó có thể kết luận rằng có sự khác biệt có ý nghĩa thống kê trong cảm nhận giữa các nhóm ứng viên có thâm niên công tác khác nhau về Sự thu hút của tổ chức đối với họ, hay nói cách khác thì cảm nhận về tính hấp dẫn của tổ chức đối với các nhóm ứng viên tuyển dụng có thâm niên công tác khác nhau là khác nhau.

\section{Kết luận và khuyến nghị}

Kết quả nghiên cứu cho thấy cả 3 yếu tố danh tiếng tổ chức, tương thích con người công việc và tương thích con người - tổ chức đều tác động đến Sự thu hút của tổ chức đối với các ứng viên tuyển dụng. Trong đó, danh tiếng tổ chức có tác động mạnh nhất (0.391) đến khả năng thu hút của bệnh viện Nhân dân Gia Định. Kết quả xếp hạng đánh giá chất lượng bệnh viện trong 2 năm gần nhất là 2017 và 2018 do Sở Y tế Thành phố Hồ Chí Minh công bố cho thấy bệnh viện Nhân dân Gia Định lần lượt nằm ở vị trí thứ 2 và thứ 1 . Bệnh viện cũng là cơ sở thực hành chủ yếu cho các trường đại học lớn như Đại học $\mathrm{Y}$ dược Thành phố Hồ Chí Minh, Đại học Y khoa Phạm Ngọc Thạch, Đại học Nguyễn Tất Thành. Ngoài ra, bệnh viện cũng là nơi tiếp nhận đào tạo cho các bệnh viện tuyến dưới. Chính vì sự phổ biến, những thông tin tốt từ bệnh viện đã lan tỏa và tạo cho bệnh viện khả năng thu hút các ứng viên từ chính sự nổi tiếng này. Chính vì vậy, bệnh viện cần xác định bản thân là ngọn cờ đầu trong khu vực y tế công để luôn phấn đấu giữ vững vị thế nhằm thu hút các ứng viên tiềm năng phục vụ cho sự nghiệp chăm sóc sức khỏe.

Tương thích Con người - Tổ chức có tác động đáng kể đến khả năng thu hút của tổ chức (0.274). Bệnh viện Nhân dân Gia Định hiện tại là một bệnh viện công lập, do đó bản thân bệnh viện luôn mang trong mình sứ mệnh công là cải thiện sức khỏe nhân dân. Chính việc không đặt doanh thu là một tiêu chí hàng đầu trong hoạt động đã tạo ra được cảm giác phù hợp của các ứng viên phù hợp với các giá trị và hình ảnh của bệnh viện. Ngoài ra, bệnh viện hiện đang thực hiện theo cơ chế tự chủ tài chính sẽ có nhiều kinh phí hơn trong việc thực hiện các chính sách đãi ngộ, đào tạo, thu hút nhân tài, lương thưởng. Môi trường bệnh viện bao gồm các chuyên gia hàng đầu cũng sẽ là một trường học lớn để các nhân viên y tế hoàn thiện bản thân nhiều hơn. Nhằm hỗ trợ cho các ứng viên xác định sự phù hợp khi nộp đơn xin việc, bệnh viện cần công khai những nét nổi bật của bệnh viện đến với các ứng viên tuyển dụng như văn hóa tổ chức, 
quy tắc ứng xử, nội quy lao động, hệ thống khen thưởng - kỷ luật, những quyền lợi khi trở thành nhân viên chính thức.

Tương thích Con người - Công việc là yếu tố có tác động đến sự thu hút của bệnh viện (0.192). Bệnh viện Nhân dân Gia Định hiện có tất cả 48 khoa, phòng. Trong đó, có 30 khoa lâm sàng, 8 khoa cận lâm sàng và 10 phòng chức năng. Chính cơ cấu khoa, phòng đầy đủ, đa dạng chuyên khoa đã tạo ra nhiều vị trí việc làm phù hợp với nhiều đối tượng ứng viên. Các ứng viên khi dự phỏng vấn sẽ có được cảm giác tương thích với công việc khi hiểu được vai trò, vị trí của họ trong tổ chức. Tuy nhiên, tác động của việc tương thích công việc đến khả năng thu hút của tổ chức là thấp hơn hẳn so với danh tiếng tổ chức và tương thích con người - công việc. Bệnh viện Nhân dân Gia Định cần lưu ý nâng cao cảm nhận phù hợp với công việc của các ứng viên tuyển dụng thông qua xây dựng cụ thể hơn bảng mô tả công việc, mô tả năng lực, bổ sung những nội dung phỏng vấn gợi mở những hoài bão, dự định tương lai trong công việc của các ứng viên nhằm xây dựng mô tả vị trí việc làm phù hợp cũng như cải thiện các chế độ, chính sách khi thấy cần thiết. Hơn thế nữa, bệnh viện có thể xây dựng các buổi kiến tập trước khi tiến hành phỏng vấn nhằm tạo cho ứng viên những cái nhìn chung nhất và đưa ra đánh giá của cá nhân về sự phù hợp với công việc đang ứng tuyển.

Phân tích phương pháp ANOVA cho thấy không có sự khác biệt trong cảm nhận của các nhóm ứng viên có trình độ khác nhau đã phản ánh vẩn đề ở khâu tuyển dụng chính là chưa thực sự tạo điểm nhấn cho các nhóm nhân lực trình độ cao. Để tạo được sự khác biệt nhằm lôi cuốn những cá nhân có chuyên môn giỏi, đặc biệt là các chuyên gia đầu ngành thì công tác tuyển dụng cần nêu rõ những ưu đãi, các cơ hội phát triển, môi trường làm việc cho các ứng viên y tế chất lượng cao hoặc dự phỏng vấn vào các vị trí việc làm khó tuyển thông qua trao đổi trực tiếp hoặc niêm yết tại thông báo tuyển dụng.

Kết quả phân tích dữ liệu cũng chỉ ra rằng có sự khác biệt về cảm nhận của các nhóm ứng viên có thâm niên khác nhau về sự hấp dẫn của tổ chức. Hiện tượng này xảy ra do sự luân chuyển nhân sự ở mức cao ở các giữa các sở y tế. Cá nhân có thâm niên công tác càng cao thì càng quen thuộc với các chính sách có phần tương tự lẫn nhau giữa các cơ sở y tế dẫn đến giảm đi cảm giác hào hứng của họ đối với một bệnh viện khác. Ngược lại, các nhóm ứng viên càng có ít thâm niên công tác thì trải nghiệm của họ không thật sự đa dạng sẽ có hứng thú với những môi trường mới lạ, những trải nghiệm, viễn cảnh đang mở ra đối với họ. Để khắc phục được điều này, bệnh viện cần thực hiện cả 2 chính sách thu hút song song cho các đối tượng ứng viên có thâm niên công tác khác nhau.

- Đối với các ứng viên có thâm niên công tác ít hoặc chưa có: Bệnh viện có thể xây dựng thêm các sân chơi dành cho nhân viên y tế trẻ có thâm niên công tác dưới 3 năm. Các cơ hội thăng tiến cũng cần được bình đẳng hơn với các nhân viên mới nhằm giữ nhân đội ngũ nhân lực chất lượng cao.

- Đối với các ứng viên đã có thâm niên công tác cao: Bệnh viện có thể tạo cho họ nhiều hơn các cơ hội tiếp xúc với các chuyên gia đầu ngành thông qua các hội thảo, tập huấn trong và ngoài nước. Những cơ hội trong học tập, thăng tiến, khen thưởng cũng cần được lưu ý để tạo sự công bằng cao nhất trong bệnh viện. 


\section{Hạn chế và hướng nghiên cứu tiếp theo}

Bên cạnh những kết quả đã thu thập được. Nghiên cứu tồn tại một số hạn chế cần phải có các nghiên cứu tiếp theo để bổ sung và hoàn thiện hơn trong việc tìm hiểu về Sự thu hút của tổ chức.

Thứ nhất, Dữ liệu của nghiên cứu thu thập cắt ngang trong khoảng thời gian 09/2018 đến $01 / 2019$. Kết quả nghiên cứu có thể thay đổi khác nhau tại các thời điểm nghiên cứu khác nhau. Các nghiên cứu tiếp theo cần có thời gian thu thập dữ liệu dài hơn để phản ánh rõ kết quả nghiên cứu.

Thứ hai, Sự thu hút của tổ chức ngoài 3 yếu tố là Danh tiếng tổ chức, Tương thích Con người - Công việc, Tương thích Con người - Tổ chức có thể nhiều yếu tố khác có khả năng tác động. Các nghiên cứu tiếp theo cần khám phá thêm các yếu tố khác vào mô hình các yếu tố tác động.

Thư $b a$, Nghiên cứu được thực hiện tại bệnh viện Nhân dân Gia Định. Do đó, kết quả nghiên cứu có thể không phù hợp khi áp dụng tại các cơ sở y tế công lập khác. Các nghiên cứu tiếp theo có thể mở rộng hơn phạm vi nghiên cứu ở khu vực y tế công tại Thành phố Hồ Chí Minh hoặc xa hơn nữa là so sánh sự khác biệt trong khả năng thu hút ứng viên tuyển dụng tại khu vực y tế công và tư tại Thành phố Hồ Chí Minh.

\section{Tài liệu tham khảo}

Acarlar, G., \& Bilgiç, R. (2013). Factors influencing applicant willingness to apply for the advertised job opening: The mediational role of credibility, satisfaction and attraction. The International Journal of Human Resource Management, 24(1), 50-77.

Aiman-Smith, L., Bauer, T. N., \& Cable, D. M. (2001). Are you attracted? Do you intend to pursue? A recruiting policy-capturing study. Journal of Business and Psychology, 16(2), 219-237.

Babakus, E., Yavas, U., \& Ashill, N. J. (2010). Service worker burnout and turnover intentions: Roles of person-job fit, servant leadership, and customer orientation. Services Marketing Quarterly, 32(1), 17-31.

Cable, D. M., \& Judge, T. A. (1996). Person-organization fit, job choice decisions, and organizational entry. Organizational Behavior and Human Decision Processes, 67(3), 294-311.

Cable, D. M., \& Judge, T. A. (1997). Interviewers' perceptions of person-organization fit and organizational selection decisions. Journal of Applied Psychology, 82(4), 546-561.

Cable, D. M., \& Turban, D. B. (2003). The value of organizational reputation in the recruitment context: A brand-equity perspective. Journal of Applied Social Psychology, 33(11), 2244-2266.

Carless, S. A. (2005). Person-job fit versus person-organization fit as predictors of organizational attraction and job acceptance intentions: A longitudinal study. Journal of Occupational and Organizational Psychology, 78(3), 411-429. 
Carless, S. A., \& Wintle, J. (2007). Applicant attraction: The role of recruiter function, worklife balance policies and career salience. International Journal of Selection and Assessment, 15(4), 394-404.

Carpenter, D. (2010). Reputation and power: Organizational image and pharmaceutical regulation at the FDA. Princeton, NJ: Princeton University Press.

Chapman, D. S., Uggerslev, K. L., Carroll, S. A., Piasentin, K. A., \& Jones, D. A. (2005). Applicant attraction to organizations and job choice: A meta-analytic review of the correlates of recruiting outcomes. Journal of Applied Psychology, 90(5), 928-944.

Chatman, J. A. (1989). Improving interactional organizational research: A model of personorganization fit. Academy of management Review, 14(3), 333-349.

Christensen, R. K., \& Wright, B. E. (2011). The effects of public service motivation on job choice decisions: Disentangling the contributions of person-organization fit and personjob fit. Journal of Public Administration Research and Theory, 21(4), 723-743.

Collins, C. J., \& Han, J. (2004). Exploring applicant pool quantity and quality: The effects of early recruitment practice strategies, corporate advertising, and firm reputation. Personnel Psychology, 57(3), 685-717.

Dineen, B. R., Ash, S. R., \& Noe, R. A. (2002). A web of applicant attraction: Personorganization fit in the context of web-based recruitment. Journal of Applied Psychology, 87(4), 723.

Fombrun, C. J., Gardberg, N. A., \& Sever, J. M. (2000). The reputation quotient SM: A multistakeholder measure of corporate reputation. Journal of Brand Management, 7(4), 241255.

Fortune. (1993). America's most admired corporations. FORTUNE Magazine, 127(3), 44-53.

Gomes, D., \& Neves, J. (2011). Organizational attractiveness and prospective applicants' intentions to apply. Personnel Review, 40(6), 684-699.

Grady, P. (2010). Internal branding, employee branding. In The IABC handbook of organizational communication (pp. 231-240).

Hair, J. F., Black, W. C., Babin, B. J., Anderson, R. E., \& Tatham, R. L. (2006). Multivariate data analysis. Upper Saddle River, NJ: Pearson Prentice Hall.

Harris, M. M., \& Fink, L. S. (1987). A field study of applicant reactions to employment opportunities: Does the recruiter make a difference? Personnel Psychology, 40(4), 765-784.

Highhouse, S., Lievens, F., \& Sinar, E. F. (2003). Measuring attraction to organizations. Educational and Psychological Measurement, 63(6), 986-1001.

Honeycutt, T. L., \& Rosen, B. (1997). Family friendly human resource policies, salary levels, and salient identity as predictors of organizational attraction. Journal of Vocational Behavior, 50(2), 271-290.

Judge, T. A., \& Cable, D. M. (1997). Applicant personality, organizational culture, and organization attraction. Personnel Psychology, 50(2), 359-394.

Kristof, A. L. (1996). Person-organization fit: An integrative review of its conceptualizations, measurement, and implications. Personnel Psychology, 49(1), 1-49. 
Kristof-Brown, A. L., Zimmerman, R. D., \& Johnson, E. C. (2005). Consequences of individuals' fit at work: A meta-analysis of person-job, person-organization, persongroup, and person-supervisor fit. Personnel Psychology, 58(2), 281-342.

Lawler, E. E., Kuleck, W. J., Jr., Rhode, J. G., \& Sorensen, J. E. (1975). Job choice and post decision dissonance. Organizational Behavior and Human Performance, 13(1), 133-145.

Liu, B., Liu, J., \& Hu, J. (2010). Person-organization fit, job satisfaction, and turnover intention: An empirical study in the Chinese public sector. Social Behavior and Personality: An International Journal, 38(5), 615-625.

Madera, J. M. (2012). Using social networking websites as a selection tool: The role of selection process fairness and job pursuit intentions. International Journal of Hospitality Management, 31(4), 1276-1282.

Mowday, R. T., Porter, L. W., \& Steers, R. M. (1982). Employee-organizational linkages: The psychology of commitment, absenteeism, and turnover. Chicago, IL: University of Chicago Press.

Neuman, L. W. (2007). Social research methods: Quantitative and qualitative approaches (6th ed.). Boston, MA: Allyn \& Bacon.

Nguyen, T. D. (2012). Phương pháp nghiên cưu khoa hoc trong kinh doanh [Scientific research method in business]. Hanoi, Vietnam: Nhà xuất bản Lao động - Xã hội.

Perez, E. M., Walton, A. L., Cooper, D. M., \& Pacheco, M. R. (2014). Unpacking organizational attraction: A process model. International Journal of Business and Social Science, 5(10), 108-120.

Phillips, J. M., Gully, S. M., McCarthy, J. E., Castellano, W. G., \& Kim, M. S. (2014). Recruiting global travelers: The role of global travel recruitment messages and individual differences in perceived fit, attraction, and job pursuit intentions. Personnel Psychology, 67(1), 153-201.

Ralston, S. M., \& Brady, R. (1994). The relative influence of interview communication satisfaction on applicants' recruitment interview decisions. The Journal of Business Communication (1973), 31(1), 61-77.

Rynes, S. L., Bretz, R. D., Jr., \& Gerhart, B. (1991). The importance of recruitment in job choice: A different way of looking. Personnel Psychology, 44(3), 487-521.

Saks, A. M., \& Ashforth, B. E. (1997). A longitudinal investigation of the relationships between job information sources, applicant perceptions of fit, and work outcomes. Personnel Psychology, 50(2), 395-426.

Smither, J. W., Reilly, R. R., Millsap, R. E., Pearlman, K., \& Stoffey, R. W. (1993). Applicant reactions to selection procedures. Personnel Psychology, 46(1), 49-76.

Taylor, F. W. (1911). The principles of management. Manhattan, NY: Harper \& Brothers.

Turban, D. B., \& Cable, D. M. (2003). Firm reputation and applicant pool characteristics. Journal of Organizational Behavior, 24(6), 733-751.

Van Hoye, G. (2012). Recruitment sources and organizational attraction: A field study of Belgian nurses. European Journal of Work and Organizational Psychology, 21(3), 376-391. 
Vien An (2017). Top các bệnh viện có điểm chất lương cao nhất tại TP.HCM [Top hospitals with the highest quality scores in HCMC]. Retrieved December 20, 2018, from https://thanhnien.vn/suc-khoe/top-cac-benh-vien-co-diem-chat-luong-cao-nhat-taitphcm-790191.html

Werbel, J. D., \& Gilliland, S. W. (1999). Person-environment fit in the selection process. In N.W. Schmitt (Ed.), Handbook of personnel selection and assessment (pp.252-273).

Yen Nhi (2019). Sở Y tế TPHCM công bố 'bảng xếp hạng' bệnh viện [HCM City Department of Health announces 'ranking' hospitals] . Retrieved December 20, 2018, from https://www.tienphong.vn/suc-khoe/so-y-te-tphcm-cong-bo-bang-xep-hang-benh-vien1366146.tpo 